\title{
Author Correction: The immuno-oncological challenge of COVID-19
}

Lisa Derosa, Cléa Melenotte, Franck Griscelli, Bertrand Gachot, Aurélien Marabelle, Guido Kroemer (D) and Laurence Zitvogel (D)

Correction to: Nature Cancer https://doi.org/10.1038/s43018-020-00122-3, published online 2 October 2020.

In the version of this article initially published, there was an error in Table 3. In the row for Oseltamivir, in the fifth column "Antitumor effect in vivo," the text now reading "Pro-tumorigenic effects in mice: Increased aggressiveness of canine mammary tumor xenografts ${ }^{276 \text { " }}$ initially appeared as "In canines and mice: Increase mammary tumor aggressiveness ${ }^{276}$." The change has been made to the HTML and PDF versions of the article.

Published online: 8 February 2022

https://doi.org/10.1038/s43018-022-00342-9

(c) Springer Nature America, Inc. 2022 